\title{
De la expresividad a la teatralidad, Schönberg, Kurtag y otros
}

\author{
From expresivity to theatrality, Schönberg, Kurtag and others \\ Jesús Arreguín Zozoaga ${ }^{a}$
}

\begin{abstract}
:
This text is a reflection on some aspects related to the aesthetic process of vocal music, which were changing their parameters over the different historic periods in art, such as Barroque, Classic, Romantic and particularly the Twentieth Century, based on the aesthetic view of some composers. This, by revising some representative works of that century, which have, at the same time, served as a parameter for recent generations of our century, and which through the years, have evolved changing the paradigms of tradition and some of their aspects. As an example of this, the reinterpretation of the exacerbated expression, in a technical projection, which nourished from the gesture or has profited of it in order to surpass its borders, that is, intending to fusion music and theater, since their linkings have always been evidenced, one next to the other, but not linked as it is possible now, in a same discourse beyond the musicality of poetry or the own immanence of word rhythm.
\end{abstract}

\section{Keywords:}

Expressivity, gesture, theatricality, corporality and interdisciplinarity

\section{Resumen:}

Este texto es una reflexión sobre algunos aspectos relativos al proceso estético de la música vocal, que fueron cambiando sus parámetros a través de los diferentes períodos históricos en el arte, como el Barroco, Clásico, Romántico y en particular el siglo XX, a partir de la estética de algunos compositores. Esto a través de algunas obras representativas de ese siglo que a su vez han servido como parámetro para las recientes generaciones de nuestro siglo, y que, en el transcurso del tiempo, a su vez, han evolucionado cambiando los paradigmas de la tradición y algunos de sus conceptos. Prueba de ello es, por ejemplo, la reinterpretación de la expresión exacerbada, en una impostación técnica que se nutrió de la gestualidad o se ha servido de ella para rebasar sus fronteras, esto es, intentar fundir la música con el teatro, pues siempre se había tenido en claro su comunión una al lado de la otra, pero no fusionadas cómo es posible en la actualidad, en un mismo discurso más allá de la musicalidad de la poesía o la inmanencia propia del ritmo en la palabra.

\section{Palabras Clave:}

Expresividad, gestualidad, teatralidad, corporalidad e interdisciplanariedad

\section{Introducción}

Tomando la premisa de que todo fluye y se transforma, la estética musical también se desarrolla, principalmente a partir de los conceptos teóricos y de las posibilidades de ejecución instrumental y vocal, que eventualmente, alcanzaron niveles de virtuosismo o maestría generadas a finales del Barroco y en el Clacisimo para el lucimiento del intérprete de talento como para la nobleza misma (depositaria de las buenas maneras y virtudes así como de sus polisibilidades adquisitivas en diversos rubros), como por ejemplo el famosísimo Farinelli a quién su propio hermano Riccardo Broschi, le escribía para su lucimiento como en Qual guerriero in campo armato, Aria de la ópera Idaspe, entre otros compositores como Nicola Porpora; o la conocidísima Aria de La Reina de la Noche de Las Bodas de Fígaro de Mozart. Un proceso de evolución como el aludido, se fundamenta al menos en dos factores, en primer lugar el desarrollo del arte musical en sí mismo, el cual conlleva las habilidades técnicas instrumentales de los músicos, como las propuestas innovadoras de los propios compositores y en segundo lugar, por la necesidad de nuevos recursos expresivos

\footnotetext{
a Jesús Arreguín Zozoaga; Profesor Investigador del Instituto de Artes de la Universidad Autónoma del Estado de Hidalgo. Contacto:
} 
que revaloran la tradición y aportan una nueva perspectiva a la estética vigente (Luciano Berio trabajó estrechamente con intérpretes de alto nivel para la confección de sus famosas Secuenze para instrumentos solos), donde también entra en función el contexto y desarrollo filosófico y humanista evidenciado a través de la literatura y la poesía. En el contexto del ámbito vocal, la relación entre Schiller y Beethoven son el más clásico de los ejemplos con la Oda a la Alegría de la 9na. Sinfonía, que desembocara posteriormente en la base de los postulados de la "Ilustración" en la Francia de finales del siglo XVIII.

Es en la música de cámara donde la voz comienza sutilmente a liberarse de su anterior proyección masiva y mediante una especie de introspección, de la austeridad de un canto más íntimo que va más a la conciencia personal y reflexión, va surgiendo la exaltación a la belleza de la naturaleza, del amor y del espíritu a través de los himnos y el patriotismo en los tiempos de crisis por las revultas y eventuales guerras en el orbe. Así, se abandona paulatinamente la convención del virtuosismo -que en su tiempo fue también crisol de la perfecciónpara cimentarse a través de los años en otra percepción de la belleza, ahora dotada de otra realidad social y más general mediante la emotividad del canto y sobre todo del dramatismo de las pasiones humanas cristalizadas en la ópera del siglo XIX con Puccini y Verdi, entre muchos más.

La gran maquinaria de la ópera mueve las grandes pasiones y viene una especie de renacimiento de la tragedia, para hacer más evidentes las fuerzas del mundo, de la vida 0 del destino, plasmadas fundamentalmente en lo concerniente a la consumación sublimada del amor. Con Wagner se había regresado parcialmente al ámbito mítico de los súper-hombres, semidioses y dioses, pero su música incorporó otros requerimientos para la emisión vocal, la potencia y resistencia física como elementos constitutivos de un drama más allá del libreto y de la pasión trasladada del texto a la música; esto es, la acción dramática completa con todos los elementos de la ópera puestos en juego casi al límite de sus posibilidades -incluyendo el tiempo de desarrollo de estas- que confrontaron la estética del Bel canto, hasta la corrosión misma del sistema armónico al que sólo podía escampar el concepto como un símbolo. Después de Wagner es evidente la gran influencia de Schönberg -como estafeta- en el desarrollo de la música del siglo $\mathrm{XX}$, no sólo por la transformación que de ella hizo, sino por el rigor con el que lo realizó: de la exacerbación armónica a su corrupción, de ahí a su posterior extinción o anulación (atonalidad) y finalmente a una nueva sistematización mediante la Dodecafonía.
Veamos pues, una visión global del proceso de transformación de la estética vocal en la música.

\section{Desarrollo}

Una de las articulaciones de la técnica vocal que quedó plasmada en su escritura y constituyó una pauta en la composición musical, fue sin duda la generada por Schönberg en sus categorías de Sprechstimme y Sprechgesang -surgidas en los tiempos del expresionismo- generando un énfasis natural o un tipo de exacerbación de la expresividad musical. Según Méndez (2012), Rousseau pensó que "toda música extrae su carácter principal de la lengua que es propia de su contexto social, en particular de su contexto nacional. Sería la prosodia de la lengua la principal responsable del carácter musical propio de una sociedad". Con el tiempo, muchos compositores abordaron ese tipo de emisión vocal, derivando incluso en una teatralidad más allá de las necesidades naturales de la ejecución instrumental y más cercana al teatro mismo.

Es posible que para Stockhausen y Berio, la nueva percepción del mundo vaticinada por la postura estética de Schönberg a partir de la atonalidad y la poética expresionista quasi onírica del Pierrot Lunaire (1912); los acontecimientos generados por los futuristas y postulados de Marinetti, los ismos periféricos del dadaísmo y lo "concreto", así como sus propias experiencias en la Segunda Guerra Mundial como adolescentes, haya detonado otras maneras de hacer música y potenciado incluso la transfiguración de la tradición vocal. La experiencia de la electrónica llegó para ambos compositores en su plenitud cuando estaban terminando de formarse fuertemente en la tradición, sin embargo, ante los acontecimientos culturales, políticos y bélicos que envolvían su desarrollo, constituyó una parte muy importante de ella. Era el tiempo de dar paso a otra poética y por ende estética del arte, como habían promulgado los futuristas tres décadas antes.

El Pierrot Lunaire, de Schönberg fue fundamental en este proceso de transformación a partir de su propuesta estética al plantear categorías distintas de canto mediante tipos diversos de impostación de la voz. A lo largo de su propia obra, el compositor hizo la desarticulación, la suspensión de jerarquías y paradigmas, y la reagrupación y estructuración de una nueva estética musical. Un proceso análogo del inicial modus operandi de la música electrónica de las décadas de los 40's a los 60's., el cual puso en relieve Stockhausen con Gesäng der Jünglinge (1956) -piedra angular de la música electrónica- pocos años después de haber iniciado su trabajo en el Estudio de Música 
Electroacústica de la Radio Oeste de Colonia NWDR y WDR en 1955, curiosamente, la pieza revela la manipulación vocal de manera evidente con textos distorsionados y en sentido contrario a la emisión natural, y aunque este tratamiento había ya sido realizado por su "inventor" Pierre Schaeffer, en la Radio Televisión Francesa (RTF), a partir de grabaciones de trenes, con su estudio Étude aux chemins de fer (1948) y con la Symphonie pour un homme seul (1950) en colaboración con Pierre Henry; el tratamiento de estas obras "concretas" no estaba aún en el rigor profundo de la nueva estética, aunque si como una gran novedad y nuevo punto de partida, para ese nivel, fue necesaria la pragmática estructuración musical de Stockhausen.

Es sintomática la cita que hace Stockhausen del Pierrot, una década después, acaso como un homenaje en la parte central de su obra vocal Stimmung (1968), de largo discurso que emula con nuevas articulaciones y modos de emisión vocal, sonoridades y ruidos de parámetros muy semejantes a los generados por medios electrónicos; adelantándose -o siendo a su vez visionarioa otros procesos basados en la tecnología, semejante a la que fue génesis de ésta misma obra (osciladores y moduladores de onda, frecuencia y voltaje, etc.), y a otros compositores como los franceses del I'tineraire, de los años 70's con su propuesta espectral de la música. No obstante, un lustro atrás había desarrollado ya la novedad vocal del Pierrot con Momente (1962-64), para Soprano, cuatro grupos corales y trece instrumentistas, en donde la solista interactúa entre los instrumentistas y los cantantes, como un puente tímbrico intermitente o emulador de ambas categorías de producción sonora a partir del desarrollo e innovación de la articulación vocal.

El compositor inglés Bryan Ferneyhough realizaría también una especie de homenaje a Schönberg con su obra On Stellar Magnitudes (1994), en la cual emplea la misma dotación instrumental del Pierrot Lunaire, cambiando en la instrumentación la soprano por mezzosoprano.

otro sentido y más directamente relacionado con la dramaturgia de la obra, es el empleo que hace del Sprechystimme el compositor polaco Krzysztof Penderecki, en su ópera de gran potencia dramática Los demonios de Loudun (1969). Pero desde el punto de vista del virtuosismo vocal solista, la voz es llevada a otro límite con la Secuenza III (1966) para voz sola de Luciano Berio, cuyo empleo vocal linda con la ejecución de tipo instrumental en figuraciones rítmico-melódicas y exploraciones tímbricas en función del tipo de articulación, sonora, gestual y lingüística, constituyéndose en una pieza obligada en el ámbito de la música vocal contemporánea por el alto grado de requerimientos técnicos para su ejecución, basados en la apertura mental, resistencia física y control musical que aporta otra perspectiva semiótica. Como argumentan Galicia \& Orozco (2013), "El hecho que el lenguaje no siempre sea fiable para generar unos significados precisos en la mente de otra persona es un reflejo de su fuerza como medio para crear nuevas comprensiones".

En Italia, el ambiente de modernismo pujante fue encabezado por Berio y Maderna, quienes habían incursionado en la electrónica con Ritratto di Città (1954) a mediados de los años 50's, en el Estudio de Fonología y Música Electrónica en Milán. La vanguardia también fue representada con Nono y su evidente postura política comunista y de cambio contestatario en varias de sus composiciones, algunas de las cuales integran a la instrumentación la sonoridad de voces, ruidos o efectos heredados de la técnica de grabación a megáfono o mediante los aparatos de las estaciones radiofónicas captadas en cinta magnética, relacionada de manera paralela a la denominada Música Concreta de Schaeffer, donde el material sonoro era modificado mediante el correr a distintas velocidades de las cintas a carrete abierto de las máquinas de grabación modificando también su altura, así como con descargas de voltaje variado y de manera aleatoria, etc. La técnica de este tipo de estética, elaboración musical o composición a partir de la incorporación de varios materiales sonoros, mezclados y transformados de múltiples maneras y conformando manualmente -mediante el "cortar y pegar"- la fijación de la pieza en la cinta, incluso a partir de la fragmentación o "descomposición" del material mediante la interacción de sonidos generados artificialmente por los osciladores y sintetizadores modulares y el equipo de audio utilizado en las estaciones de radiocomunicación, pudo también haber influido en Berio en un sentido conceptual y permeado parcialmente su manera de hacer música, al incorporar elementos culturales o de otros países e incluso sugerir mediante articulaciones silábicas 0 fonemas, lenguas extrañas y no una temática concreta como el caso particular el segundo movimiento $O$ King, de su de Sinfonía (1968-69), entre otras obras. Pues "No lejos de lo imperfecto de esas cualidades del ruido se encuentran las del mundo de la psique, desde la nebulosa propia del imaginario hasta la de la propia sustancia psicológica, cuya manifestación sin disimulo revela la hondura laberíntica de los tonos emocionales." (Estrada citado en Dallal, 2006, p.156)

Ligeti, probablemente impostó sus experiencias y técnicas de la música electrónica a la expresión coral con 
Lux Aeterna (1966) para 16 voces a capella, pues es evidente el empleo de cambios de color durante el desarrollo de la obra como si se tratara de la aplicación de filtros de frecuencia o supresores de ruido a la obra.

Un caso particular lo reviste la obra del compositor Mauricio Kagel, quien abordó un amplio espectro de la música a través de la escena, para reinterpretar en la mayoría de sus obras el campo semántico de la música. Un ejemplo de esto es su ópera experimental Staatstheater (1967-70), que repercutió incluso en la obra de varios compositores actuales y que bien puede contextualizarse como neo dada o teatro del absurdo; los pocos instrumentos tradicionales como el caso de una guitarra tienen una connotación arcaica y es empleada también para una canción popular a dúo y un tango (que alude la identidad del compositor), único momento en que se emplea la ejecución instrumental de manera tradicional y sin pretensiones técnicas. En términos generales, hay dos categorías sonoras en escena, una a partir de grabaciones y la otra mediante la producción de ruidos o efectos de las múltiples articulaciones del instrumental empleado, de hecho, la gran variedad de objetos empleados son personajes principales de la obra en un complejo alegórico y conceptual, que parece cuestionar el estado del arte en esos momentos, a través de la manifestación interdisciplinaria aún vigente y planteada como la negación de la ópera, por un teatro instrumental.

A finales de la década de los 70's, hubo un entusiasmo generalizado respecto al empleo de la voz o idiomática del canto y varios compositores le aportaron una fisonomía distinta en algunas de sus obras. Ligeti fue de los primeros que llevó la expresión vocal a su categoría instrumental en ensamble debido al alto grado de virtuosismo ya sea como obbligato o concierto para voz, manteniendo un intrincado dialogo con la parte orquestal con su "anti-anti ópera" El Gran Macabro (1975-78), obra desarrollada a partir de la percepción que tuvo de la ópera experimental Staatstheater de Kagel, a la que hace alusión en la obertura donde realiza una especie de coral con bocinas o cornetas ${ }^{*}$ de bicicletas que se mezclaran en una textura de metales; Kagel las empleó para evidenciar el andar en cuatro extremidades de una persona (en la primera cuarta parte de la obra), sin embargo, con esta obra Ligeti llevó el estado del arte musical a otro nivel más de exigencia.

György Kurtág, en la segunda mitad del siglo, arremete a los logros precedentes de la voz con el dúo KafkaFragmente (1976-1985) y con Mensajes de la fallecida señorita R. V. Troussova (1976-1980) con la voz articulada instrumentalmente como personaje con relieve individual, en el complejo sonoro de una orquesta de cámara donde convergen avances previos de la técnica vocal con una nueva perspectiva idiomática, como el Sprechgstimme por ejemplo.

El compositor griego Georges Arpeghis desarrolló una perspectiva de la expresividad musical, diferente a la concepción de la teatralidad musical emanada naturalmente como parte de las necesidades expresivas en la ejecución, aplicándola al teatro y fusionándola en un binomio entrelazado, esto es, música-teatro y teatromúsica, derivando de la música lo teatral como del teatro lo musical. En este ámbito, muchas de sus obras se inclinan más sobre la representación escènica que da relieve al sentido teatral, sin embargo, aunque estén dotadas de cierta dramaturgia esta puede impactar conceptualmente más a un público general, no obstante al preciosismo virtuosista de lo musical. Les sept crimes de lámour (1979) para soprano, clarinete y percusión (zarb), es una obra muy divertida, con preeminencia de la articulación vocal, rica sonoramente, dotada de cierta sensualidad y de direccionalidad, representa bien el concepto artístico del compositor, hacer teatro de todo, pero la música no se dirige sobre sus propios caminos, es comparsa o complemento de algo más, que abre o enriquece sin duda otras posibilidades expresivas en un "nuevo" tipo de género dentro de lo interdisciplinario. Como argumenta Ussa (2013), "La conciencia, en la actitud gestual favorece la adecuada expresividad y, en consecuencia, la coherencia del discurso. [...] a su vez, se convierte en espacio donde el comportamiento cognitivo de los interlocutores es el reflejo de sus estados de conciencia".

Es importante hacer referencia a ciertas composiciones que anteceden varios de sus trabajos y sobre los cuales se puede tejer un hilo conductor, por ejemplo en Récitations № 11 (1977-78), pone a prueba la capacidad del intérprete para elaborar un discurso e impostación de un personaje, sólo mediante la articulación de fonemas veloces que con su cadencia pueden confundirse con algún idioma o lengua desconocida para quien escucha (hecho que Berio empleó en varias obras cuya síntesis es La Secuenza III para voz establecida una década atrás). Mora \& Asuaje (2012), comentan que en la República de Platón, se diserta sobre el ritmo y modulación, asociando la música con el habla en cuanto a las variaciones en tono e inflexiones de la voz. "Asumía el filósofo, además, que los diferentes modos musicales o prosóidíai (PROS\%DIAI) imitaban las diferentes maneras de hablar".

\footnotetext{
* Que son empleadas como guía de acciones o correcciones de manera cómica por los payasos.
} 
De manera paralela En formes de récitations (i?), puede interpretarse también como el proceso de manejo y proyección de la voz tanto como la presencia en el escenario. Puede percibirse un método de memorización de manera lúdica mediante la reminiscencia de juegos y rondas infantiles en las que se van adicionando fonemas o palabras a las precedentes hasta el máximo posible, algo semejante al fundamento del juego Simon: dispositivo en el que se activa un sonido y enciende un cuadrante de color a manera "propuesta" y el jugador imita como "respuesta", de ser correcta esta, se amplía la secuencia a repetir, el jugador repite y así sucesivamente hasta que el proceso termine o si el jugador se equivoca se reinicia el juego. La pieza Retrouvailles (2013), performance para dos percusionistas puede tener como antecedente a ?Corporeil (1985) de Vinko Globokar, en la que explora los sonidos del cuerpo humano al ser percutido por "uno mismo", así como las sonoridades posibles de emisión bucal como resonador y sin emisión propiamente musical, gemidos y articulaciones de baja intensidad en ruido como el empleo de letras consonantes y ciertos balbuceos. Globokar compositor contextualizado dentro de la "música experimental" emplea mucho la improvisación y parámetros de indeterminación como factores de libertad para el ejecutante, estuvo cercano como intérprete en algunos proyectos de composición, como la Secuenza V (1965) para trombón de Berio; Pas de cinq (1965) para cinco actores paseantes de Kagel y Aus den sieben Tagen (1967-68) para cualquier formación instrumental de Stockhausen.

Evidentemente varias obras de muchísimos compositores -patrimonio histórico del arte musicalirradian su influencia y algunas han dejado una huella tan profunda que son reconocibles en otras por su gesto, patrones rítmicos, melódicos y armónicos casi al instante; merced a esa fuerza o particularidad que las caracteriza han sido objeto de cita por otros compositores que por diferentes razones las emplean para elucidar algún concepto en particular de sus propias propuestas estéticas y hacer $o$ no, referencia $u$ homenaje al compositor que estableció dicho gesto en la literatura musical. Por ejemplo, el Concierto de Aranjuez (1939) para guitarra de Joaquín Rodrigo, ha permeado gran parte de la literatura para ese instrumento en distintas latitudes, pues no pocos compositores pueden sustraerse a èl, ¿es acaso una cita obligada, una conveniencia o no se la pueden quitar de la memoria? Como dijera Ricaurte (2014), "Los distintos tipos de memoria (por su naturaleza, duración, contenido, forma de procesamiento) muestran la indisoluble conexión entre lo individual y lo social y la manera en que los procesos cognitivos se encuentran asociados a procesos sociales".
Las "referencias" a ciertas obras pueden ser por motivaciones vagas o pueriles y de conveniencia, como por la seducción de la música misma. Un ejemplo muy banal de esto es el tema musical de John Williams para el film Jaws (1975) de Steven Spielberg, fuertemente emparentado con The augurs of spring, de $\mathrm{La}$ Consagración de la Primavera de Stravinski. Es una práctica común de varios compositores para cine que impostan estilos como los de Wagner, Ravel, etcétera, para los denominados soundtrack fílmicos. Por supuesto existen las coincidencias y no es de dudar que sean momentos de sincronicidad en la inspiración y procesos del desarrollo creativo en determinadas obras de varios creadores, que confluyen en latitudes y tiempos distintos.

A este respecto se puede hacer referencia a coincidencias 0 influencias estéticas como entre Stockhausen y Berio mediante Momente y la Secuenza III, respectivamente, o a la coincidencia conceptual entre aspectos como el tiempo y ritmo entre Nancarrow y Ligeti a juicio de este último al conocer su obra para pianola, así como las influencias o coincidencias en su obra Clocks and Clouds (1972-1973), con ciertos aspectos de la obra de Reich y Riley. Así mismo, es posible pensar en cierta influencia de Stockhausen hacia Meredith Monk, o fuente de inspiración para esta última a partir de Stimmung, en donde ciertos giros melódicos y repeticiones aparecen, aunque metamorfoseados, como característica generalizada en su música fundamentalmente vocal.

En el caso particular de estos tres autores norteamericanos -incluyendo además a Phillip Glass- es posible rastrear la tradición ancestral de la música de muchas culturas basada en la repetición y patrones rítmicos sencillos, pero con un fuerte arraigo al inconsciente y a lo más íntimo del Hombre, el pulso corporal, es tan ontológico como la experiencia sonora y rítmica de los instrumentos de percusión en donde una sencilla secuencia y poderío textural en un ensamble bastan por su energía, para capturar a quién escucha tal fenómeno. Estos procedimientos repetitivos evocan situaciones rituales y extáticas prácticamente arquetípicas.

\section{Conclusiones}

Evidentemente todo evoluciona con el tiempo, las necesidades generan los medios para renovar y dar un nuevo impulso a todo aquello que ya se ha sistematizado con anterioridad; se irradia a las distintas manifestaciones artísticas propias de la cultura, permeando sus formas expresivas y conceptos al grado de impregnarse conceptualmente de manera paralela al avance de la 
tecnología. En la música, el canto evolucionó tanto como la técnica instrumental situándose en el umbral de una potencial hibridación con el arte dramático con quien siempre compartió una especie de simbiosis, pero aproximadamente desde hace 50 años apuntó a una síntesis al grado de no poderse disociar una disciplina de la otra. Como se advirtió ya, esto sólo se verifica en algunas obras y de ciertos compositores en particular, pues como todo, es necesario mucho tiempo de adecuación estética para que una novedad vaya más allá y se convierta en algo generalizado. Siempre han existido obras experimentales o que están adelantadas a su tiempo, para lo cual es menester una maduración social y artística que permita que los espacios físicos y mentales que aún ocupan otro tipo de manifestaciones o representaciones, posibiliten la interacción con esas propuestas "nuevas" que no pocas veces son centenarias ya, y que con el paso del tiempo tan largo para darles cabida, no pocas veces terminan eclipsadas.

\section{Referencias}

Arpeghis, G. En formes de récitations. (¡?). Recuperado el 12 de octubre de 2016 desde: https://www.youtube.com/watch?v=HGYOw81 wil0

Arpeghis, G. Les sept crimes de lámour. (1979). Recuperado el 12 de octubre de 2016 desde: https://www.youtube.com/watch?v=aZ48kO_LiRs

Arpeghis, G. Récitations $\mathrm{N}^{\mathrm{o}}$ 11. (1977-78). Recuperado el 12 de octubre de 2016 desde: https://www.youtube.com/watch?v=cgDM-pyMqxw

Arpeghis, G. Retrouvailles. (2013). Recuperado el 12 de octubre de 2016 desde: https://www.youtube.com/watch?v=03LFmAf_DM

Berio, L. O King y su Sinfonía. (1968-69). Recuperado el 12 de octubre de 2016 desde: https://www.youtube.com/watch?v=4xnonVCjKwc

Berio, L. Secuenza III. (1965). Recuperado el 12 de octubre de 2016 desde: https://www.youtube.com/watch?v=1hxjCIANddU

Berio, L. Secuenza V (Recuperado el 12 de octubre de 2016 desde: https://www.youtube.com/watch?v=ZqlUhN7TbAk

Berio, L. \& Maderna, B. Ritratto di Città, Studio per una rappresentazione radiofonica. Recuperado el 12 de octubre de 2016 desde: https://www.youtube.com/watch?v=CGCtmv_E5zE

Dallal, A. (2006). El proceso creativo, XXVI Coloquio Internacional de Historia del Arte, UNAM (Instituto de Investigaciones Estéticas), México.
Ferneyhough, B. On Stellar Magnitudes. (1994). Recuperado el 12 de octubre de 2016 desde: https://www.youtube.com/watch?v=5aTZVILfPtQ

Galicia, M. \& Orozco, G. (2013). Valoración de la semántica en la comprensión de palabras orales, Revista Chilena de Neuropsicología, 8 (2), pp. 27-30. Recuperado 16 de septiembre de 2015 desde: www.redalyc.org/articulo.oa?id=179329660001

Globokar, V.? Corporeil. (1985). Recuperado el 12 de octubre de 2016 desde: https://www.youtube.com/watch?v=QtZeUznYIj0

Kagel M. Pas de cinq. (1965). Recuperado el 12 de octubre de 2016 desde: https://www.youtube.com/watch?v=-BqZILDP95E

Kagel M. Staatstheater. (1967-70). Recuperado el 12 de octubre de 2016 desde: https://www.youtube.com/watch?v=IuM8sYuZPp8

Kurtag, G. Kafka-Fragmente. (1976-1985). Recuperado el 12 de octubre de 2016 desde: https://www.youtube.com/watch?v=2VVGqdgaxPk

Kurtag, G. Mensajes de la fallecida señorita R. V. Troussova. (1976-1980). Recuperado el 12 de octubre de 2016 desde: https://www.youtube.com/watch?v=6jQyE99SANM

Ligeti, G. El Gran Macabro. (1975-78). Recuperado el 12 de octubre de 2016 desde: https://www.youtube.com/watch?v=uS5-A61Ow4s

Ligeti, G. Clocks and Clouds. (1972-1973). Recuperado el 12 de octubre de 2016 desde: https://www.youtube.com/watch?v=vElpoSC7a2A

Ligeti, G. Lux Aeterna. (1966). Recuperado el 12 de octubre de 2016 desde: https://www.youtube.com/watch?v=iVYu5lyX5M

Méndez, A. (2012). Mejor que el significado. El umbral simbólico entre sonido y sentido, Revista Faro, 15 pp.1-15. Recuperado el 12 de septiembre de 2015 desde: https://dialnet.unirioja.es/descarga/articulo/4160155.pdf

Mora, E. \& Asuaje, R. (2011), El canto de la palabra: una iniciación al estudio de la prosodia. (pp.17-33), Venezuela: ww.2.ula.ve/publicacionesacademico. Recuperado el 26 de septiembre del 2015 desde: http://www.serbi.ula.ve/serbiula/librose/pva/Libros\%20de\%20PVA\%2 0para\%20libro\%20digital/prosodia.pdf

Penderecki, K. Los demonios de Loudun. (1969). Recuperado el 12 de octubre de 2016 desde: https://www.youtube.com/watch?v=tefsoyY-nJA

Ricaurte, P. (2014). Hacia una semiótica de la memoria, En-claves del Pensamiento, 8 (16), pp.31-54. Recuperado el 12 de septiembre de 2015 desde: http://www.redalyc.org/articulo.oa?id=141132947002 
Rodrigo, J. Concierto de Aranjuez. (1939). Recuperado el 12 de octubre de 2016 desde: https://www.youtube.com/watch?v=yeFvKCZp3s

Schaeffer, P. Étude aux chemins de fer. (1948). Recuperado el 12 de octubre de $2016 \quad$ desde: https://www.youtube.com/watch?v=N9pOq8u6-bA

Schaeffer, P. \& Henry, P. Symphonie pour un homme seul. (1950). Recuperado el 12 de octubre de 2016 desde: https://www.youtube.com/watch? $v=N 9 p O q 8 u 6-b A$

Schönberg, A. Pierrot Lunaire. (1912). Recuperado el 12 de octubre de 2016 desde: https://www.youtube.com/watch?v=bd2cBUJmDr8

Stockhausen, K. Aus den sieben Tagen. (1967-68). Recuperado el 12 de octubre de 2016 desde: https://www.youtube.com/watch?v=e9nJIY4o710

Stockhausen, K. Gesäng der Jünglinge. (1956). Recuperado el 12 de octubre de 2016 desde: https://www.youtube.com/watch?v=Y1Psx24n3rM

Stockhausen, K. Stimmung. (1968). Recuperado el 12 de octubre de 2016 desde: https://www.youtube.com/watch?v=3hPkJW95jsw

Stockhausen, K. Momente. (1962-64). Recuperado el 12 de octubre de 2016 desde: https:/www.youtube.com/watch?v=wkA_XFus78

Stravinsky, I. La Consagración de la Primavera. (1913). Recuperado el 12 de octubre de 2016 desde: https://www.youtube.com/watch?v=BhsRshwB5q4

Ussa, M. (2013). Semántica gestual y comunicación humana, Cuadernos de Lingüística Hispánica, 21, pp. 89-10. Recuperado 16 de septiembre de 2015 desde: http://www.redalyc.org/articulo.oa?id=322227529007

Williams, J. Jaws. (1975). Recuperado el 12 de octubre de 2016 desde: https://www.youtube.com/watch?v=lV8i-pSVMaQ 